\title{
Comprendiendo la Economía Venezolana: Petróleo y dependencia externa (1960-2017)
}

Medardo Alfonso Palomino Arias Universidad Antonio Nariño Colombia 


\title{
Comprendiendo la Economía Venezolana: Petróleo y dependencia externa (1960-2017).
}

\author{
Medardo Alfonso Palomino Arias (DD \\ Universidad Santiago de Cali - Colombia
}

Para citaciones: Palomino, M. (2020). Entendiendo la Economía Venezolana: Petróleo y dependencia externa (1960-2017). Panorama Económico, 28(3), 143-158. https://doi.org/10.32997/pe-2020-2697

Recibido: 23/06/2019

Aprobado: 17/10/2019

Autor de correspondencia: Medardo Alfonso Palomino Arias medardoalfonsopalomino@gmail.com

Editor: Andrés Escobar Espinoza Universidad de Cartagena, Colombia.

Tipología IBN Publindex: Artículo de Reflexión

Copyright: (c) 2020. Palomino, M. Este es un artículo de acceso abierto, distribuido bajo los términos de la licencia https://creativecommons.org/licenses/bync-sa/4.0/ la cual permite el uso sin restricciones, distribución y reproducción en cualquier medio, siempre y cuando que el original, el autor y la fuente sean acreditados.

\section{RESUMEN}

El presente artículo examina la economía venezolana a través del análisis del producto agregado, por el lado de la demanda y por el lado de la oferta. En función de la disponibilidad de datos, se emplean distintas fuentes en un horizonte temporal variable, que oscila entre 1960 y 2017 . Se evidencia que la crisis económica tiene causas estructurales, donde subyace un sector externo que impone límites al crecimiento económico. Durante la Revolución Bolivariana, la economía venezolana se ha caracteriza por crecer con base al consumo y las exportaciones. Por otro lado, su inversión mostró una fuerte dependencia de las importaciones que, a su vez, dependen de la mono-exportación de petróleo. Por tanto, el modelo basado en el consumo interno se agota, ante la ausencia de cambios en la estructura productiva que permitan sustituir la renta petrolera.

Palabras clave: Macroeconomía; Crecimiento económico; Dependencia petrolera.

JEL: B22, O4.

\section{Understanding venezuelan economy: Oil and foreign dependence (1960-2017).}

\section{ABSTRACT}

This article analyzes the aggregate production, considering the demand and the supply axes within Venezuelan economy. Depending on the availability of data, different sources are used for the period 1960 and 2017. It is evident that the economic crisis has structural causes, where there is an underlying external sector that imposes limits on economic growth. During the Bolivarian Revolution, Venezuelan economy has been characterized by growing based on consumption and exports. On the other hand, its investment showed a strong dependence on imports which, in turn, depend on the mono-exports of oil. Therefore, the model based on internal consumption is exhausted, in the absence of changes in the productive structure that allow replacing oil revenues.

Key words: Macroeconomics; economic growth; oil dependency.

JEL: B22, O4. 


\section{INTRODUCCIÓN}

Los avatares que afectan a la economía venezolana son un tema importante por sus implicaciones económicas, políticas y sociales para la región latinoamericana y los países en desarrollo que dependen de la explotación de recursos naturales no renovables, y muy particularmente, para las naciones cuyo sector externo depende, en gran medida, de la exportación de petróleo. Para el caso de Venezuela, durante la segunda década del siglo XXI, el país ha presentado una importante caída de la producción agregada que merece ser analizada.

Para tal fin se estudia la economía venezolana en el periodo de los años 1960 y 2017, con el fin de identificar los problemas estructurales que explican la crisis económica. Para este propósito, se realiza un análisis del producto venezolano empleando series de tiempo para examinar los componentes del Producto Interno Bruto (PIB), por el lado de la demanda y por el lado de la oferta. Se considera como referente el inicio del gobierno de Hugo Chávez en 1999, o la denominada Revolución Bolivariana.

El artículo se divide en 4 secciones. La primera sección, muestra los problemas estructurales del crecimiento económico venezolano. La segunda sección, se presenta desde una perspectiva teórica el funcionamiento de una economía cuyo sector externo tiene gran relevancia. Posteriormente, en la tercera sección se exploran los componentes del PIB por el lado de la demanda, con el propósito de identificar las variables que jalonaron al conjunto de la economía. En la cuarta sección, se examina las tendencias del PIB por el lado de la oferta, mostrando el comportamiento de los grandes sectores económicos para analizar la estructura productiva del país. Por último, se presentan las conclusiones.

\section{La crisis de Venezuela}

Lander (2018) argumenta que Venezuela hace parte de los países exportadores de naturaleza, en donde se ha configurado un petroestado, en que la riqueza depende, en mayor medida, de la exportación de petróleo, en lugar de la generación de valor por medio del trabajo. Por tanto, el modelo económico del país se ha desarrollado con base en el ingreso que proviene de la naturaleza, condición que no cambió con el advenimiento de la Revolución Bolivariana, muy por el contrario, se acentuó, al aumentar la dependencia de la renta petrolera. A partir de la contabilidad nacional, el ingreso venezolano es mayor a su producto, mostrando así que en el país impera un capitalismo rentístico que crece gracias, como lo afirma Baptista (2005), a "un excedente rentístico, cuyo carácter internacional implica que nada le cuesta a la economía producirlo" (p.106), lo que lo vuelve inviable. De la misma manera, Kornblihtt (2017) encuentra que la crisis económica radica en su estructura de país dependiente de la renta de la tierra petrolera, de manera que la sobrevaluación de la tasa de cambio es insostenible en el tiempo. De tal forma, la crisis sería una manifestación de un modelo no productivo sino rentístico, que esa afectada cuando caen los precios internacionales del petróleo, que evidencias todas sus contradicciones.

La conceptualización de Kornblihtt (2017) sirve para entender que el capital nacional tiene interdependencia con el capital mundial, en donde coexiste una industria petrolera que provee de ingreso a una industria no petrolera que se mantiene en funcionamiento 
gracias a su conducta de captadora de renta. Tal captura de renta por parte de la burguesía, que cambia bolívares por dólares, se obtiene gracias a la sobrevaluación de la moneda que produce el auge petrolero (compra de divisas relativamente baratas). Tal sobrevaluación, como mecanismo de apropiación de la renta petrolera ha sido transversal, independiente de la Revolución Bolivariana. En este sentido, los capitales dentro del país pueden operar con una productividad por debajo de la productividad media mundial ${ }^{1} y$, de acuerdo con Baptista (2005), incluso pagar salarios reales por encima de su productividad, en la medida en que dure el boom petrolero y la renta fluya para evitar el ajuste que provoca la crisis.

Vera (2018) señala que la crisis global venezolana es explicada por un conjunto de factores que se engloban en tres dimensiones: crisis externa, crisis productiva y crisis fiscal. En su planteamiento, la crisis externa da origen a una crisis de deuda, que ha crecido en tiempos de bonanza, pero con el declive de los precios internacionales del petróleo, el servicio a la deuda se torna problemático. En ese contexto, el gobierno central tomó la decisión de hacer el ajuste macroeconómico a través de la reducción de las importaciones, lo que genera cuellos de botella para el aparato productivo importador de bienes intermedios y bienes de capital. Por otro lado, se afirma que Venezuela sufre una guerra económica, en donde los mayores niveles de escasez coinciden con eventos electorales entre 2003 y 2013 (Curcio 2015; Curcio, 2016) y se asegura que la inflación que experimenta el país no tiene fundamentos económicos, ni se trata de un fenómeno monetario.

La razón radicaría en un ataque a la economía venezolana, por parte del sector adverso al chavismo, que emplea la tasa de cambio paralela a través de portales web como mecanismo para inducir la inflación. Este argumento se evidencia con el comportamiento atípico del dólar en el mercado paralelo a partir del año 2012.

Cabe señalar que el planteamiento de Curcio $(2015,2016)$ coinciden con los acontecimientos en política internacional, donde las presiones contra el gobierno de Maduro han tomado la forma de sanciones durante el gobierno del Barack Obama, y posteriormente del gobierno de Trump² (Naciones Unidas, 2018) e incluso tensiones en la frontera con Colombia (Casey, Koettl, y Acosta, 2019).

\section{Contabilidad Nacional y Planteamiento Teórico}

Desde la contabilidad nacional, la identidad macroeconómica señala que la producción total en una economía nacional puede crecer desde el lado de la demanda a través del consumo, de la inversión, del gasto público o de las exportaciones.

$$
Y=C+I+G+X-M
$$

Donde $\mathrm{Y}=$ producción, $\mathrm{C}=$ consumo de los hogares, $\mathrm{I}=$ inversión, $\mathrm{G}=$ gasto público, $\mathrm{X}=$ exportaciones, $\mathrm{M}=$ importaciones.

Una economía que busque crecer (ampliar la producción) en el corto plazo requerirá la expansión de al menos uno de los componentes, ya sea del consumo, de la inversión, del

\footnotetext{
1 Se entiende que con una moneda sobrevaluada los insumos que se adquieren del exterior tienen bajos precios relativos y los capitales operan en el mercado interno sin competir en el mercado mundial.

${ }^{2}$ Al momento de escribir este artículo, el U.S. Department of the Treasury (2019) informa sobre sanciones en materia petrolera
} contra el gobierno de Nicolás Maduro. 
gasto público o de las exportaciones, manteniendo todo lo demás constante. Sin embargo, a pesar de que las importaciones no hagan parte de la producción doméstica de la economía, bien se conoce que, en una economía abierta, la expansión de la producción requerirá, en mayor o en menor medida, la expansión de las importaciones, obedeciendo al grado de dependencia de la economía respecto a los bienes intermedios o bienes finales importados (bienes de consumo o bienes de capital).

Si las importaciones se pagan en divisas que solo se obtienen a través de la exportación de bienes ${ }^{3}$, la importación de bienes necesarios para la producción dependerá del comportamiento de las exportaciones, y sus reservas internacionales, en divisas, dependerá del tamaño del superávit obtenido en el sector externo (X-M). En otras palabras, las importaciones solo se podrán realizar siempre y cuando el país cuente con la solvencia necesaria en términos de divisas para poder importar las cantidades de bienes que su aparato productivo requiere.

En virtud de lo anterior, un país cuya producción nacional sea altamente dependiente de importaciones y experimente una caída en sus exportaciones, observará una caída en la producción al agotarse su reversa de divisas, puesto que, además de que la caída de sus exportaciones reduce de manera contable y directa la producción nacional, la falta de bienes importados impactarían negativamente el consumo, la inversión y el gasto público. Desde la teoría económica se entiende que la acumulación de capital, a través de la inversión, explica el crecimiento de la productividad de la mano de obra, la cual, partiendo de una baja proporción capital-trabajo, mayor número de bienes de capital produce más cantidad de bienes por hora de trabajo (Harrod, 1939; Domar, 1946; Solow, 1956; Kaldor, 1961). En este orden de ideas, en la identidad macroeconómica, el componente que explicaría el crecimiento, no solo de corto plazo sino también de largo plazo, sería la inversión. En la identidad bien se observa que las importaciones aportan a la inversión, de manera que aumentos de inversión requerirán aumento de las importaciones.

$I=Y-(C+G+X)+M$

Se puede afirmar entonces que, a mayor dependencia de las importaciones, mayor necesidad se tendrá de incrementos de las exportaciones para expandir la inversión, y con ello, no solo crecer en el corto plazo, sino ampliar la producción en el largo plazo. Si las exportaciones tienen un alto grado de concentración en un solo producto, la renta que proviene de la exportación del producto estrella ${ }^{4}$, es el resultado de las cantidades exportadas $(\mathrm{Q})$ por el precio internacional $\left(\mathrm{P}^{*}\right)$.

$X=P^{*} \times Q$

En este sentido, el flujo de renta exterior que contribuye a la inversión, puede mantenerse de tres formas: a) vía aumentos del precio internacional; b) vía aumento de cantidades exportadas; o c) ambas. Sin embargo, dado un país pequeño y precio aceptante, la única variable que estaría bajo su control para regular el ingreso de divisas, sería las cantidades producidas y exportadas, de manera que caídas del precio internacional podrían ser

${ }^{3}$ Los préstamos internacionales son exportaciones futuras, entendiendo que los préstamos en divisas que haga un país a otro, se sufragan en última instancia, con exportaciones que se pagan en divisas.

${ }^{4}$ Para el caso de Venezuela, el bien estrella de exportación es el petróleo. 
compensadas con aumentos en la producción del bien estrella de exportación. Lo anterior guarda relación con la necesidad de diversificación productiva que reduzca la dependencia de un solo bien de exportación. Desde el enfoque estructuralista se entiende que, para lograr la expansión de la economía y tasas altas de inversión sostenidas en el tiempo, se requiere de cambios estructurales en el aparato productivo (Ocampo, 2014). Es decir, un país que no diversifique su economía no podría mantener tasas altas de inversión en el tiempo.

Como se asegura en Ocampo (2014), el crecimiento económico es un proceso de cambio estructural donde el sector primario pierde participación en la generación de riqueza y la industria moderna y los servicios lo ganan. Lewis (1954), especificó este cambio estructural a través de un modelo de dos sectores, uno de subsistencia y el otro capitalista. En su argumentación explica el cambio productivo de las economías cuyas inversiones pasan de un sector tradicional de baja productividad a uno moderno de alta productividad relativa. Históricamente, es un patrón que se encuentra en las economías que se desarrollan (Chang 2007, 2010) y además existe evidencia de una relación entre cambio estructural y desarrollo económico (Lall, 1999; Lall, 2000; Lin y Monga, 2010).

Ocampo (2014) afirma que desde la escuela estructuralista se entiende el cambio estructural como motor del crecimiento y su relevancia radica en que la actividad industrial constituye el principal canal de transferencia del progreso tecnológico de los países del centro a la periferia, y mientras mayor diversificación de la estructura productiva haya, mayores serán las oportunidades de transferencia tecnológica y de incrementos de productividad. Desde esta visión estructuralista, las actividades primarias como la agricultura y la minería, aunque pueden experimentar incrementos de productividad, no son tan efectivas en transmitir tales incrementos al resto de sectores productivos. Por tanto, el cambio estructural muestra a una economía que crece desarrollando nuevos sectores que estimulan el cambio tecnológico.

Desde lo postulado por Lewis (1954), se puede afirmar que una economía, que se industrializa, se caracterizará porque su sector en expansión presentará altas tasas de crecimiento con respecto al resto de la economía, de manera que su participación crecerá dentro de la producción agregada y resaltará como sector líder. Murphy, Shleifer y Vishny (1989) afirman que prácticamente todo país que ha experimentado un rápido crecimiento en su productividad y en su nivel de vida, lo ha hecho a través de la industrialización, esto es, a través del incremento de sus manufacturas aprovechando economías de escala. Para casos recientes, los países del Este asiático (Lall y Teubal, 1998; Lall, 1997,1999, 2000), y en particular China (Lin, 2004, 2013; Zhu, 2012), son ejemplos de tales hechos estilizados. Con el planteamiento estructuralista se entiende que el cambio estructural del aparato productivo es condición necesaria para lograr crecimiento económico con tasas de inversión altas, y con ello, ganancias en productividad (Ocampo, 2005) y diversificación de las exportaciones (Chenery, 1975) que ayuden a superar restricciones derivadas de la necesidad de importación de bienes de capital.

\section{Producto Interno Bruto venezolano y demanda agregada}

Examinando el PIB por sus componentes de gasto en el periodo 1960-2014 (grafico 1), se encuentra que el patrón del crecimiento económico sufre un cambio a partir de 1995, cuando el índice del gasto público cae y las exportaciones y el consumo cobran relevancia 
creciendo por encima del PIB real. El gasto público solo alcanzó los niveles de los años 90 en el 2014, y su trayectoria continuó por debajo del PIB real, al igual que las importaciones y la inversión. En otras palabras, en el periodo de la Revolución Bolivariana, el país creció a partir del consumo y las exportaciones, mientras la inversión se mantuvo en un segundo plano, con un crecimiento menor al total de la economía. Tendencia que vino desde los años 80.

Gráfico 1. PIB y sus componentes

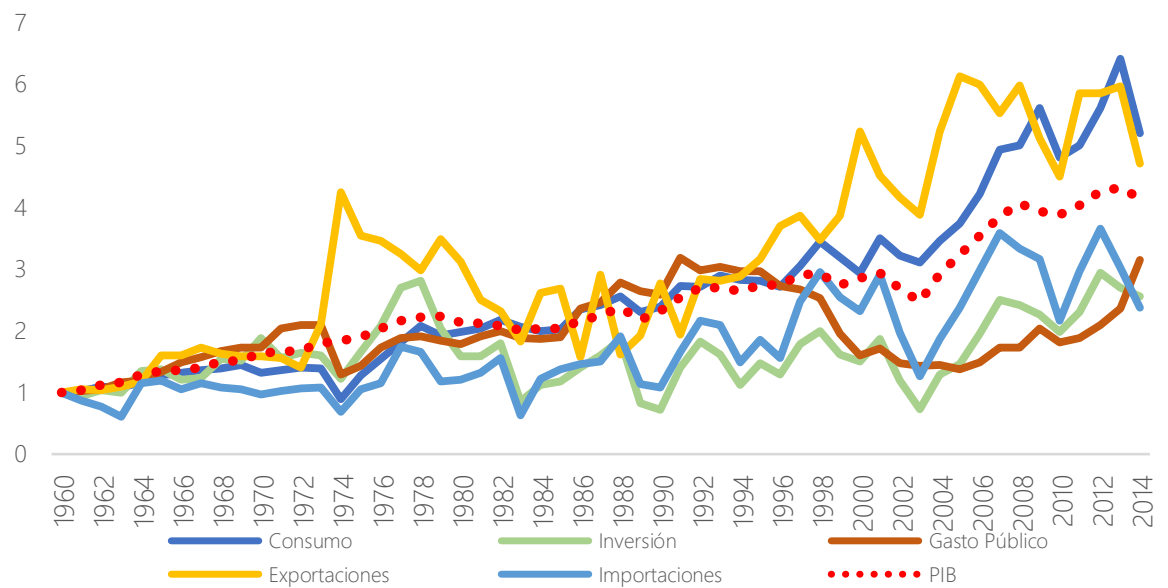

Fuente: Cálculos realizados con datos de Feenstra, Inklaar y Timmer (2015)

El grafico 2, muestra la evolución de la inversión, medida en el eje izquierdo, y la tasa de inversión (inversión/PIB), medida en el eje derecho. A pesar de las fluctuaciones a lo largo de la década 2002-2012, permaneció muy por debajo de sus registros históricos como fracción del PIB. Contrario a los casos de éxito en el Este asiático, que muestran que las tasas de inversión se mantuvieron alrededor del 30\% (Feenstra, Inklaar \& Timmer, 2015), Venezuela paulatinamente ha visto como la inversión disminuye desde su máximo de 49\% en 1978 hasta llegar a su mínimo de 11,5\% en el 2003, precedida por una fuerte caída en 1990. A pesar de una leve recuperación desde 2003, se mantiene baja para lograr altas tasas de crecimiento económico.

Gráfico 2. Dinámica de la inversión real y participación de la inversión en el PIB

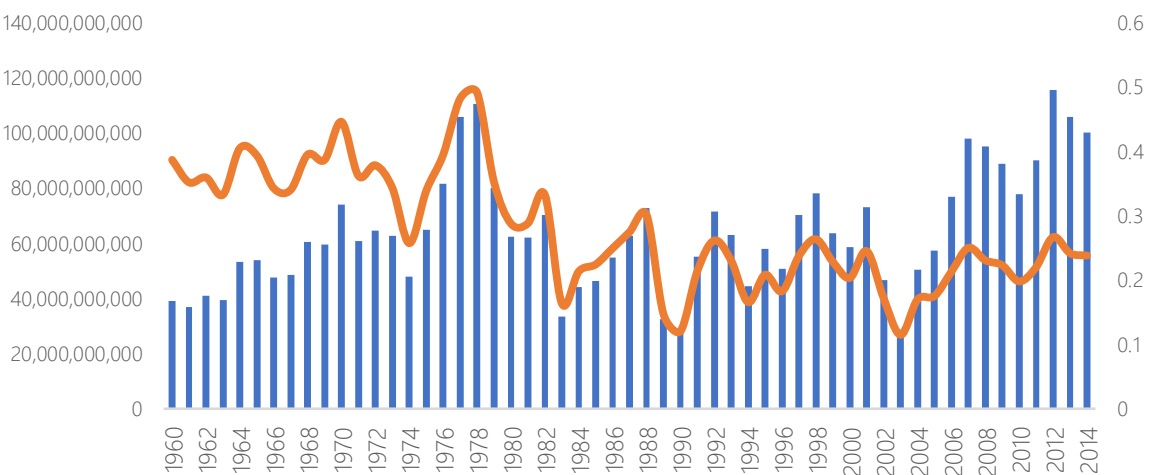

Inversión (dolares de 2010)

Inversión \%PIB

Fuente: Cálculos realizados con datos de Feenstra, Inklaar y Timmer (2015). 
En un contexto de inestabilidad política y de paro petrolero (López, 2006) en los años 2002 y 2003, la tasa de inversión pasa de un reducido 17\% a un 11,5\% respectivamente. A partir del 2004 se recupera, pero no supera el 26,6\% (su máximo en 2012), y en 2014 terminó con una tasa de inversión del 23,7\%. Por otro lado, la inversión tiene una alta correlación con las importaciones. El grafico 3, ilustra una relación positiva entre las tasas de crecimiento de la inversión y las importaciones desde 1961 hasta 2014. Ningún otro componente de la demanda agregada presenta tan alta correlación con las importaciones y el consumo en menor medida. Estos datos indicarían que el aparato productivo venezolano atiende las necesidades de consumo interno, pero requiere de la importación de bienes de capital para la ampliación de la capacidad productiva. Por lo cual, una mayor inversión requerirá mayores importaciones.

Gráfico 3. Correlación entre inversión e importaciones (1961-2014).

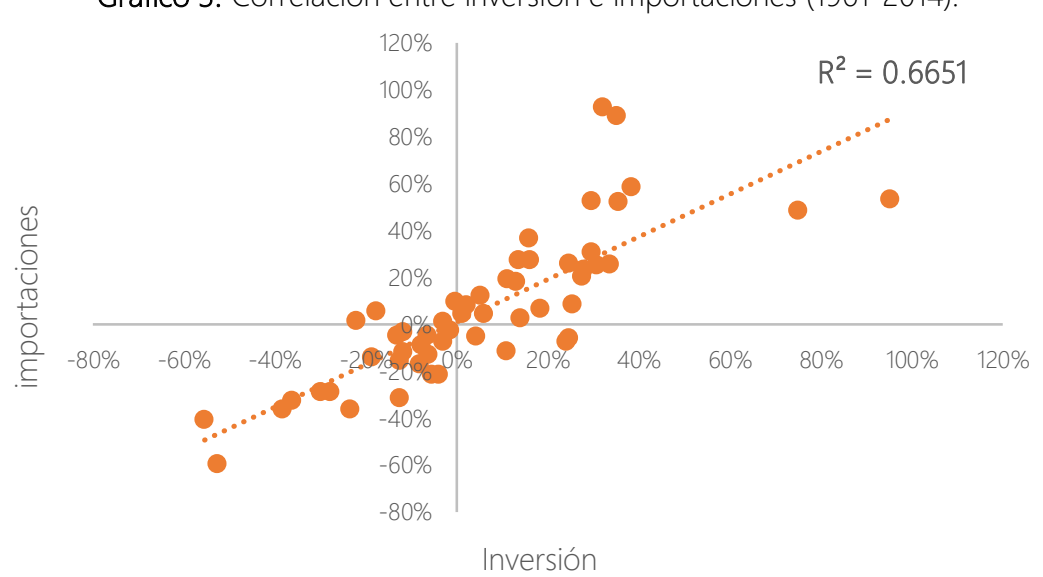

Fuente: Cálculos realizados con datos de Feenstra, Inklaar y Timmer (2015)

Sin embargo, el crecimiento del PIB a partir de la segunda mitad de los años 90 ha sido jalonado por el consumo y las exportaciones. De tal manera que el crecimiento económico depende en mayor medida de la actividad interna que sostiene el consumo y del sector externo a través de las exportaciones.

\section{Estructura productiva de Venezuela}

Con el propósito de examinar la estructura productiva venezolana (grafico 4) se descompone el PIB en los sectores que engloban el conjunto de las actividades económicas del país: Agricultura, minería, manufactura, servicios y construcción. Se observa que los sectores líderes son los servicios y la minería, muy por encima de la manufactura, la cual a partir de la segunda mitad de los años 80 presenta una tendencia decreciente en su participación en el PIB total. El comportamiento de la manufactura como porcentaje del PIB es un hecho relevante, en virtud de las experiencias de los países de la industrialización tardía, en las cuales mostró ser un sector estratégico en el crecimiento y desarrollo económico (Lall, 1997, 1999, 2000; Chang, 2007, 2010; Naudé y Szirmai, 2012). 
Gráfico 4. Evolución sectorial de la economía venezolana

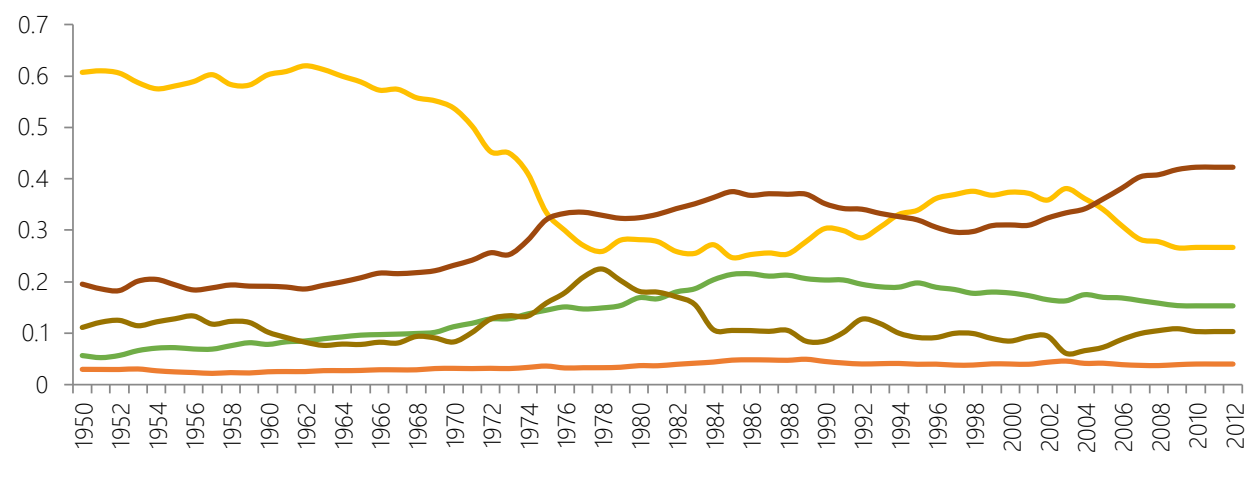

- Agricultura Minería $\longrightarrow$ Manufactura Construcción

Fuente: Datos obtenidos en Timmer, de Vries, y Vries (2015).

Planteamientos teóricos avalan la estrategia basada en la manufactura como forma de lograr el desarrollo, teniendo en cuenta los efectos de encadenamientos hacia adelante y hacia atrás (Hirschman,1958), de manera que un país que dinamiza su sector manufacturero puede aspirar a altas tasas de crecimiento económico. Para el caso de Venezuela, según Lucena y Carmona (2011), el empleo manufacturero solo creció hasta los años 80, lo cual coincide con la caída en la participación del sector en el PIB, como muestran las estimaciones de Timmer, de Vries, y Vries (2015).

Si se compara las tasas de crecimiento de la manufactura y del PIB real, se muestra el crecimiento manufacturero de largo plazo a través de la media móvil de 7 años, con el propósito de mostrar la tendencia. Desde 1961 hasta 1980, la manufactura tuvo un crecimiento constante, regularmente con tasas superiores a las del PIB real y con el mayor crecimiento de largo plazo de todo el periodo 1961-2014. Posteriormente, el crecimiento se volvió inestable, presentando variaciones porcentuales negativas, aunque con crecimientos superiores al del PIB real en algunos periodos, pero con tendencia de largo plazo decreciente.

Dentro del periodo de la Revolución Bolivariana, la manufactura tuvo crecimiento regularmente por debajo del crecimiento del PIB real, aunque se presenta la mayor tasa de crecimiento de corto plazo, 27\% en 2004. No obstante, la tendencia de largo plazo exhibe forma de u invertida, lo que muestra que no hubo recuperación sostenida de la industria manufacturera, y en 2013 tuvo la caída más pronunciada de la serie, -17\%, a pesar de que el sector era una de las apuestas en el Primer Plan Socialista (PPS) del Proyecto Nacional Simón Bolívar 5 .

${ }^{5}$ En el gobierno de la Revolución Bolivariana se conocía la importancia de lograr el cambio estructural en la matriz productiva del país a través de "sembrar el petróleo" (Lander, 2018), para lograr "la sostenibilidad del modelo" (Giordani, 2012, p.15) 
Gráfico 5. Tasas de crecimiento de la industria manufacturera y del PIB real, 1961-2014

$30 \%$

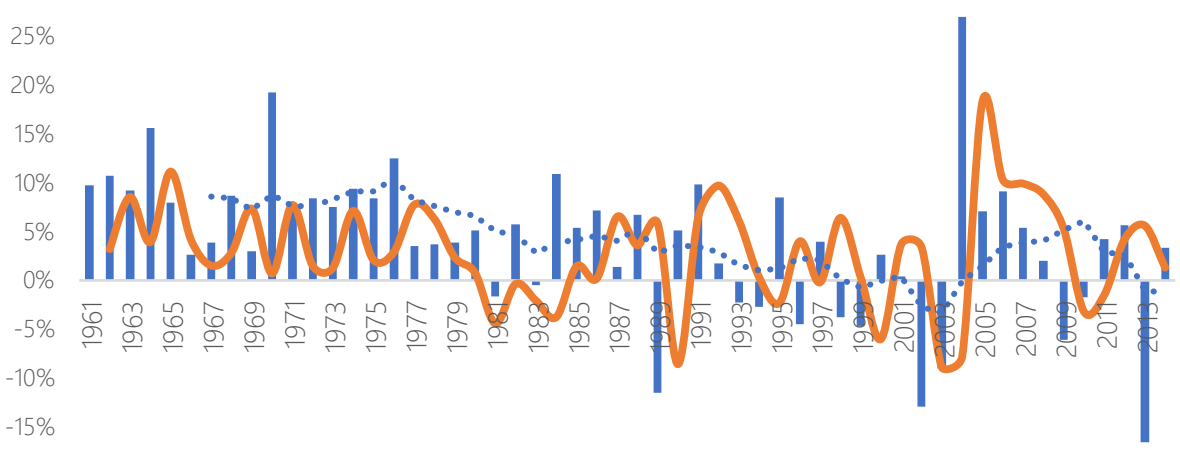

$-20 \%$

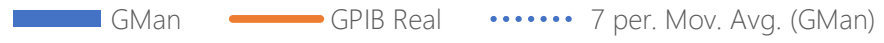

Fuente: Cálculos propios empleando datos de Timmer, de Vries, y Vries (2015) y del Banco Mundial.

Analizando las participaciones sectoriales en el PIB, se observa en la tabla 1 que los dos únicos sectores con crecimiento sostenido, acotado dentro del periodo de la Revolución Bolivariana, han sido los servicios y la construcción. Dos sectores cuya naturaleza no es de exportación ${ }^{6}$. De los tres sectores restantes, y transables, la Agricultura se mantiene con una pequeña participación alrededor del 4\%, la Manufactura y la Minería pierden participación, siendo la perdida de la segunda más grande que la de la primera. Después del sector servicios, la minería siguió siendo el sector de mayor peso.

Tabla 1. Promedios de la participación sectorial en el PIB

\begin{tabular}{lccccc}
\hline Periodo & Agricultura & Minería & Manufactura & Servicios & Construcción \\
\hline $\mathbf{1 9 9 9 - 2 0 0 2}$ & $4,1 \%$ & $36,8 \%$ & $17,4 \%$ & $31,3 \%$ & $9,0 \%$ \\
$2003-2006$ & $4,2 \%$ & $34,8 \%$ & $16,9 \%$ & $35,4 \%$ & $7,2 \%$ \\
$2007-2010$ & $3,8 \%$ & $27,3 \%$ & $15,7 \%$ & $41,3 \%$ & $10,4 \%$ \\
$2011-2012$ & $4,0 \%$ & $26,7 \%$ & $15,3 \%$ & $42,3 \%$ & $10,3 \%$ \\
\hline
\end{tabular}

Fuente: Con base en datos de Timmer, de Vries, y Vries (2015)

El sector de la minería no sólo representa una importante fracción del PIB, también de las exportaciones totales, con la mayor participación del crudo como producto estrella ${ }^{7}$ (Tabla 2). Llama la atención el gradual proceso de concentración de las exportaciones en el rubro de Productos Minerales, de los cuales la mayor parte se constituye de Petróleo Crudo. La pequeña participación relativa del petróleo refinado refleja la dependencia del país respecto a la transformación de su combustible. De acuerdo con The Observatory of Economic Complexity, el 56\% de las importaciones de petróleo refinado en el 2017 (último año con disponibilidad de datos), fueron obtenidas desde los Estados Unidos. Respecto a la industria petrolera venezolana, cabe resaltar lo siguiente. En febrero de 2002, el poder ejecutivo realiza cambios de las directivas de la empresa estatal Petróleos de Venezuela, S.A (PDVSA) (López, 2006).

${ }^{6}$ De acuerdo al Atlas of Economic Complexity, las actividades de transporte, viaje y turismo, las de mayor exportación del sector servicios, sólo representaron respectivamente el 2,08\% y 1,67\% de las exportaciones totales del 2016.

7 Helpman (2011) hace un interesante recuento sobre las teorías del comercio internacional, donde muestra los resultados de investigaciones donde la dotación factorial define el patrón de especialización del país. 
Tabla 2. Participación del sector energético en las exportaciones totales

\begin{tabular}{cccc}
\hline Año & $\begin{array}{c}\text { Productos } \\
\text { Minerales }\end{array}$ & $\begin{array}{c}\text { Petróleo } \\
\text { Crudo }\end{array}$ & $\begin{array}{c}\text { Petróleo } \\
\text { Refinado }\end{array}$ \\
\hline 1999 & $78 \%$ & $47 \%$ & $25 \%$ \\
2000 & $83 \%$ & $55 \%$ & $23 \%$ \\
2001 & $80 \%$ & $53 \%$ & $21 \%$ \\
2002 & $78 \%$ & $59 \%$ & $13 \%$ \\
2003 & $77 \%$ & $59 \%$ & $12 \%$ \\
2004 & $83 \%$ & $56 \%$ & $23 \%$ \\
2005 & $87 \%$ & $61 \%$ & $23 \%$ \\
2006 & $90 \%$ & $77 \%$ & $10 \%$ \\
2007 & $87 \%$ & $66 \%$ & $17 \%$ \\
2008 & $93 \%$ & $76 \%$ & $15 \%$ \\
2009 & $96 \%$ & $75 \%$ & $20 \%$ \\
2010 & $95 \%$ & $75 \%$ & $19 \%$ \\
2011 & $97 \%$ & $78 \%$ & $18 \%$ \\
2012 & $98 \%$ & $82 \%$ & $15 \%$ \\
2013 & $98 \%$ & $89 \%$ & $8 \%$ \\
2014 & $95 \%$ & $76 \%$ & $17 \%$ \\
2015 & $91 \%$ & $73 \%$ & $16 \%$ \\
2016 & $83 \%$ & $69 \%$ & $12 \%$ \\
2017 & $92 \%$ & $80 \%$ & $10 \%$ \\
\hline Fuente: The Observatory of Economic Complexity
\end{tabular}

Posteriormente, en diciembre de 2002 ocurre el paro petrolero, como expresión opositora al gobierno nacional, que dio lugar a la expulsión de gerentes, técnicos y trabajadores de la empresa (López, 2006; Vera, 2018). Tal expulsión de personal fue significativa, López (2006) la contabiliza en miles, aproximadamente el 45\% de los empleados de la industria, y en datos de Lucena y Carmona (2011), ascienden a 53\%. Esto significa pérdidas, no solo de activos físicos, producto del saboteo de actores políticos contrarios a proyecto de la Revolución Bolivariana, sino también pérdidas del know-how acumulado de la empresa ${ }^{8}$. El gráfico 6 muestra las tasas de crecimiento del PIB real (GPIB Real), las tasas de crecimiento de la producción de petróleo (GPP) y los precios internacionales del petróleo mediante los índices de referencia Brent y West Texas Intermediate (WTI). Estos dos índices de precios tuvieron un alza histórica en la primera década del siglo XXI, y tal auge de precios llegó hasta el año 2012 en el caso del Brent y 2013 en el caso del WTI.

Los problemas de la producción de petróleo y de las exportaciones anteceden a la caída del PIB real. En el 2002, año del paro petrolero, se observa la fuerte caída de la producción petrolera y del conjunto de la producción agregada, la cual solo se restablecería con crecimiento en el 2004. A partir del 2005 se observa la tendencia decreciente en la producción petrolera y la desaceleración del conjunto de la economía, a pesar del

\footnotetext{
${ }^{8}$ Esta pérdida de know-how implica la desaparición de conocimiento tácito adquirido a través de procesos de aprender haciendo (learning by doing), y no es susceptible de ser codificado y transmitido (ver Ancori, Bureth, y Cohendet, 2000).
} 
elevado incremento en los precios internacionales del petróleo entre 2003 y 2011, con la salvedad del año 2009.

Gráfico 6. Crecimiento económico, producción petrolera y precios internacionales del petróleo $80.0 \%$

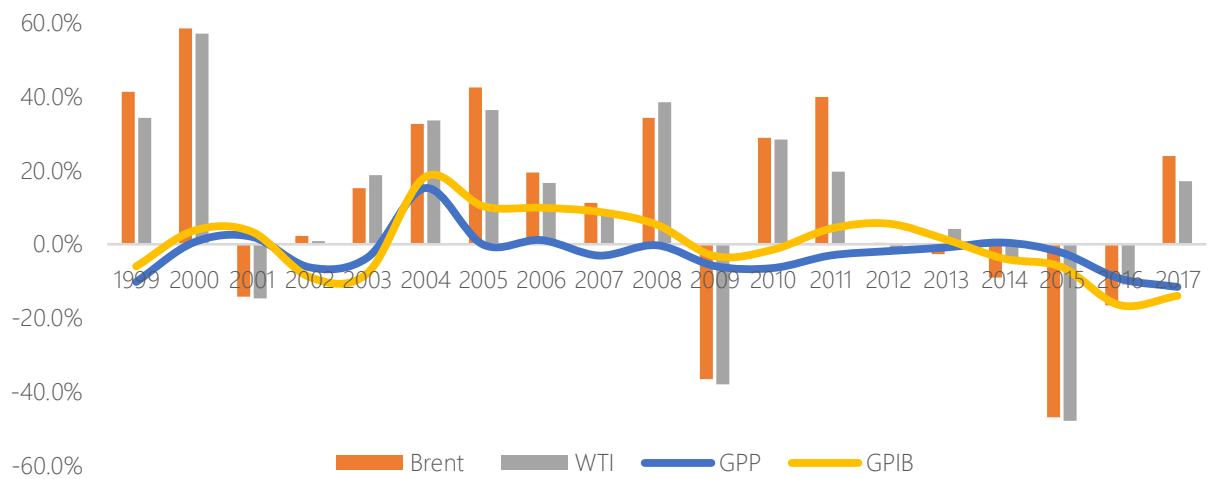

Fuente: Cálculos realizados con datos del Banco Mundial, Fondo Monetario Internacional, BP Statistical Review of World Energy.

En una situación en la que la producción petrolera no crece, los precios al alza sirven de factor compensador de la falta de aumentos en la producción, de tal forma se podrían seguir generando ingreso de divisas. Sin embargo, el modelo se agota cuando ocurre la caída de los precios internacionales del petróleo, entonces los precios ya no pueden seguir desempeñando su papel compensador. Hasta el año 2013 el país presentó tasas positivas de crecimiento, la caída de los precios internacionales del petróleo coincide con las tasas de crecimiento negativo del PIB real. Los problemas de crecimiento se extienden a otras industrias. Particular atención se merece el comportamiento sincronizado de los sectores de la industria básica y el sector azucarero.

En el periodo de la Revolución Bolivariana tuvieron sus picos máximos entre 2002 y 2008, sin embargo, si se analiza toda la serie de datos disponibles, los máximos históricos se dieron en 1970 y 1974 para el caso del petróleo y el hierro, y en 2007 para el caso del acero. La caña de azúcar tuvo su máximo en el 2003. Estas industrias, decrecen antes del choque externo ocasionado por la caída de los precios internacionales del petróleo; hasta el año 2006, la producción de petróleo y hierro crecen, para luego entran en caída libre, seguidas por la producción de acero y de caña de azúcar. El declive coincide con el Primer Plan Socialista y con una serie de nacionalizaciones que Vera (2018) fecha entre 2007 y 2009. No obstante, es necesario resaltar la crisis mundial que tomó lugar a finales del 2007, y que se convirtió en recesión en muchas partes del mundo industrializado en el año 2008 y 2009 (Tapia y Astarita, 2011). 
Gráfico 7. Producción de petróleo, de hierro y acero

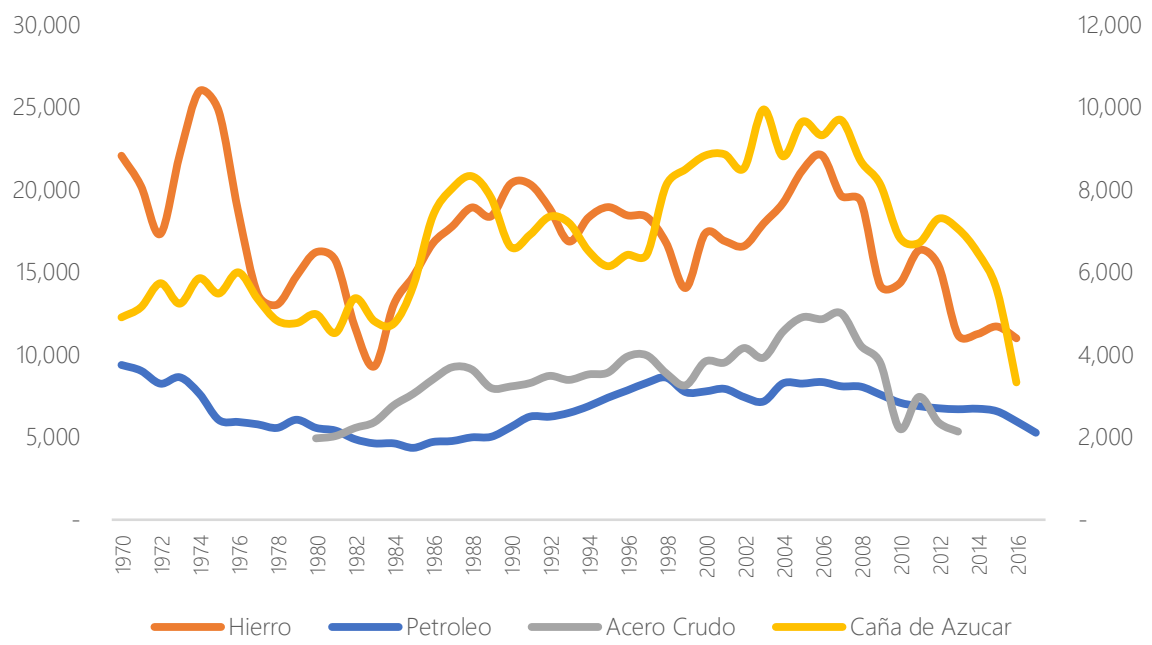

Fuente: Knoema, WorldSteel Association, BP Statistical Review of World Energy y CEPAL.

A pesar de que el Estado se hizo con el control de la industria básica y azucarera, estas reducen su producción antes del desplome de los precios internacionales del petróleo, lo que podría ser por causas de mala gestión. Lucena y Carmona (2011), afirman que tanto la industria petrolera como la del hierro fueron nacionalizadas, siendo esta ultima la segunda fuente de divisas. Vera (2018) incluye en estas nacionalizaciones a toda la industria básica, contando la del acero. También, la industria azucarera, la más importante dentro del rubro de los alimentos si se mide en toneladas producidas (según datos de la (EPAL) y cuyo mercado interno en 1999 fue abastecido casi en su totalidad por la producción nacional, fue estatizada en un 62,5\% (Transparencia Venezuela, s.f.).

El deterioro de la actividad económica en general, y del sector externo en particular, se ve reflejado en las cuentas externas con la des-acumulación de reservas internacionales. Como ilustra el grafico 8, la caída en la producción de petróleo a partir del 2007 es acompañada por el declive en las reservas internacionales ${ }^{9}$ que caen a partir de su máximo en el 2008. Por otra parte, el endeudamiento aparece como válvula de escape ante una situación de declive en la renta petrolera. En el grafico 9 ilustra la evolución tanto de las reservas internacionales como del endeudamiento a través de sus tendencias. El endeudamiento del gobierno central se encuentra expresado como porcentaje del PIB. Se observa que en el año 2008 ocurrió el punto de quiebre de la tendencia de las reservas internacionales y del endeudamiento del país. Venezuela inicia un proceso de desacumulación de reservas y endeudamiento progresivo en medio de la caída sostenida de la producción petrolera. Dada la vulnerabilidad del sector externo a los precios internacionales del petróleo, el modelo económico entra en crisis una vez disminuye la renta petrolera y se imposibilita la adquisición de bienes del exterior que permitan seguir alimentando el aparato productivo del país. De tal forma, la economía en su conjunto creció hasta el año 2013, a una tasa muy baja del 1\%, en un entorno de subida de precios del petróleo y jalonada, en gran medida, por el crecimiento del consumo, el gasto público, y en menor medida, por el crecimiento de las exportaciones.

${ }^{9}$ Las reservas internacionales están conformadas por dólares, e incluyen oro. 
Gráfico 8. Crecimiento de la liquidez y comportamiento de las reservas internacionales

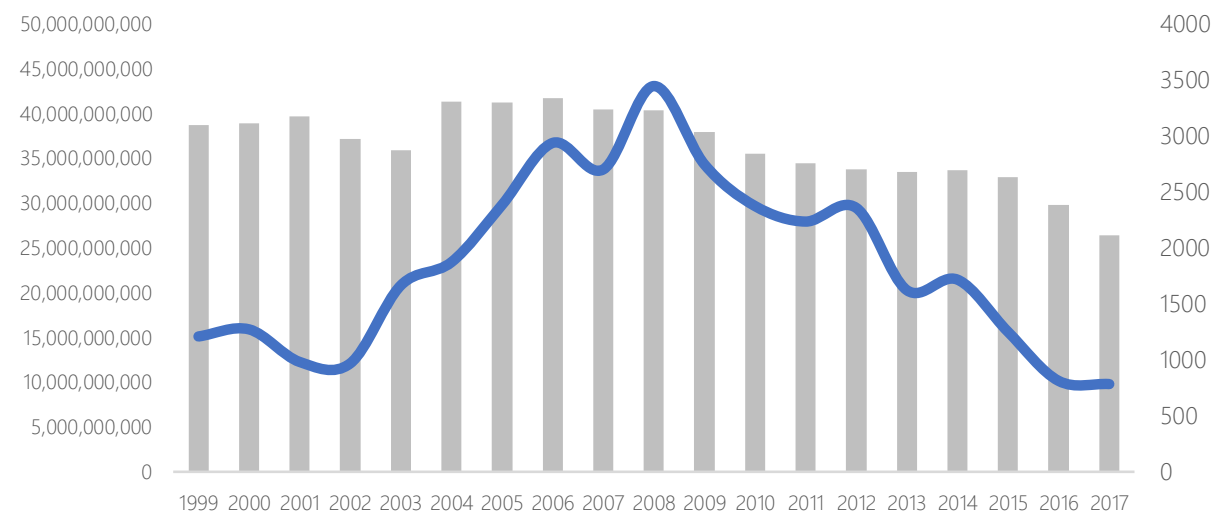

Producción Petrolera $\longrightarrow$ Reservas Internacionales

Fuente: cálculos realizados con datos del Banco Mundial y BP Statistical Review of World Energy.

Gráfico 9. Reservas Internacionales y Deuda del gobierno Central

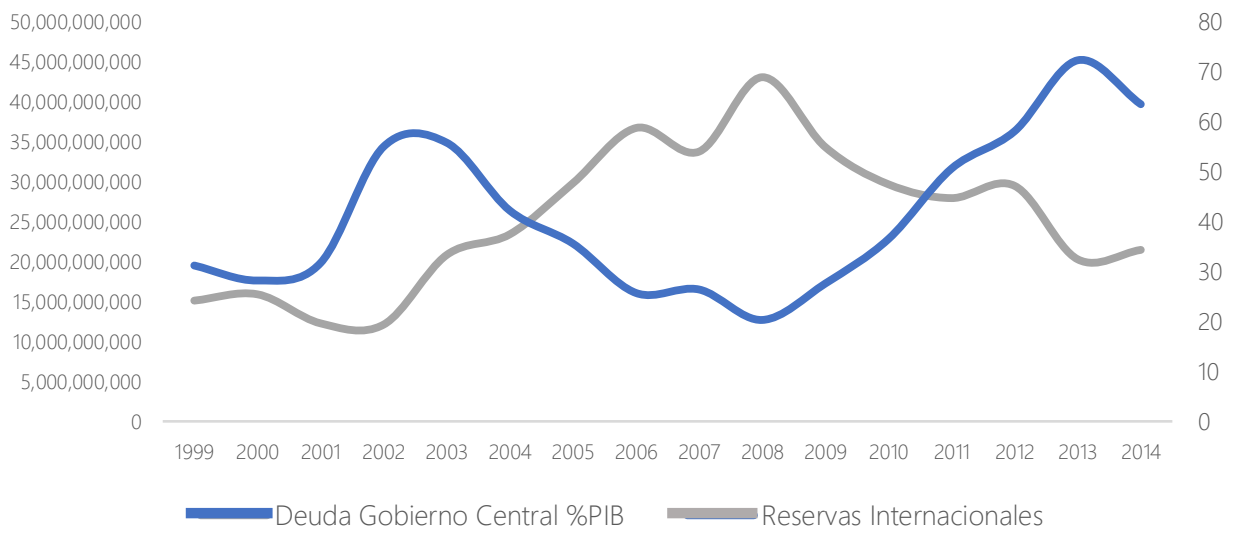

Fuente: Con base en datos del Fondo Monetario Internacional y Banco Mundial

No obstante, para ese año las importaciones cayeron junto a la inversión. Dada la dependencia de la industria domestica a las importaciones, el consumo no pudo mantener la actividad económica por mucho tiempo, y en el año 2014, cayó fuertemente y la economía en su conjunto decreció -4\% junto a la caída de los precios internacionales del petróleo, a pesar del esfuerzo del gobierno por estimular la economía a través del gasto público.

\section{CONCLUSIONES}

Como se ha evidenciado en este artículo y se ha expuesto en la literatura económica, se puede señalar que el crecimiento material venezolano, supeditado a la dependencia del capital nacional al capital mundial, pone límites al crecimiento económico y lo vuelve inviable al no desarrollar las fuerzas productivas del país. Por un lado, durante la Revolución Bolivariana la economía venezolana se ha caracteriza por crecer con base al consumo y las exportaciones. Por otro lado, su inversión mostró una fuerte dependencia de las importaciones que, a su vez, dependen de la mono-exportación de petróleo. En 
este orden de ideas, el aparato productivo del país, que en última instancia y a largo plazo, crece a través de la inversión, no puede desarrollarse sin las divisas que produce la industria petrolera. Eventualmente, el modelo basado en el consumo interno se agota, ante la ausencia de cambios en la estructura productiva que permitan sustituir la renta petrolera.

El flujo de divisas del petróleo no se tradujo en capacidad productiva, que llevara al país a depender más de la relación productiva del trabajo y del capital. Por tanto, el modelo se agotó con la caída de los precios internacionales del petróleo. Una vez entraron en caída libre las reservas internacionales, dada la dependencia de la industria domestica a las importaciones, el consumo no pudo mantener la actividad económica por mucho tiempo, es ahí que, ante la imposibilidad de sustituir la exportación de petróleo como fuente de divisas, el modelo se agotó y vino la caída de la actividad económica agregada. Todo esto, en un contexto de conflictividad política interna y externa, que, si bien tendría sus efectos, no hacen parte de las causas estructurales de la crisis del modelo rentístico venezolano.

\section{REFERENCIAS}

Ancori, Bernard., Bureth, Antoine., y Cohendet, Patrick. (2000). The Economics of Knowledge: The Debate about Codification and Tacit Knowledge. En: Industrial and Corporate Change, p. 255287

Atlas of Economic Complexity. Recuperado de: http://atlas. cid. harvard. edu/explore/?country=236\&partner=undefined \&product=undefined \&p roductClass=SITC\&startYear=undefined \&target $=$ Product \&year $=1994$

Banco Mundial. Datos de Libre Acceso del Banco Mundial. https://datos.bancomundial.org/

Baptista, Asdrúbal. (2005). El Capitalismo Rentístico, Elementos Cuantitativos de la Economía Venezolana. Cuadernos del Cendes, 60, 95-111.

BP Statistical Review of World Energy. https://www.bp.com/en/global/corporate/energyeconomics/statistical-review-of-world-energy.html

Casey, N., Koettl, C., y Acosta, D. (2019). Footage Contradicts U.S. Claim that Nicolas Maduro Burned Aid Convoy.

Comisión Económica para América Latina y el Caribe. Datos y Estadísticas. https://www.cepal.org/es/datos-y-estadisticas

Chang, H. (2007) Bad Samaritans: The Myth of Free Trade and the Secret History of Capitalism. Bloomsbury Press.

Chang, H. (2010). 23 Things They Don't Tell You About Capitalism. Allen Lane an imprint of Penguin Books. London.

Chenery, H. (1975). The Structuralist Approach to Development Policy. World Bank Reprint Series: Number Twenty.

Curcio, Pascualina. (2015). Desabastecimiento e Inflación en Venezuela. Pp. 1-43

Curcio, P. (2016). La Mano Visible del Mercado Guerra Económica en Venezuela (2012-2016), Manipulación del Tipo de Cambio e Inflación Inducida. Pp.1-36

Domar, E. (1946). Capital Expansion, Rate of Growth, and Employment.Econometrica, 14 (2), 137-147

Feenstra, R., Robert I. and Timmer, M. (2015). The Next Generation of the Penn World Table. American Economic Review, 105(10), 3150-3182,

Fondo Monetario Internacional. Data. https://www.imf.org/en/Data

Giordani, J. (2012). De la Inclusión Social a la Venezuela Productiva Bolivariana. Ediciones Correo del Orinoco.

Harrod, R.F. (1939). An Essay in Dynamic Theory. The Economic Journal, 49(193), 14-33 
Helpman, E. (2011). Understanding Global Trade. Harvard University.

Hirschman, A. (1958). The Strategy of Economic Development. New Heaven: Yale University Press.

Kaldor, N. (1961). Capital Accumulation and Economic Growth. En: King's College, Cambridge. Reprinted From The theory of Capital. Sao Paulo, Brasil: Macmillan, p. 177-222.

Knoema, World Steel Association. Sugar cane production quantity. https://knoema.com/search?query=iron\%20production\%20venezuela\&scope=visualizations\&ta $\underline{b=\text { more }}$

Kornblihtt, J. (2017). Crisis y Renta de la Tierra Petrolera en Venezuela: Crítica a la teoría de la Guerra Económica. Cuadernos del Cedes, 94, 1-30.

Lall, S. (1997). Selective Policies for Export Promotion: Lessons from the Asian Tigers. The United Nations University.

Lall, S. (1999). Competing With Labour: Skills and Competitiveness in Developing countries. International Labour Oficce Geneva.

Lall, S. (2000). The Technological Structure and Performance of Developing Country Manufactured Exports, 1985-98. Oxford Development Studies, 28(3), 337-369. DOI: 10.1080/713688318

Lall, S. y Teubal, M. (1998). "Market Stimulating" Technology Policies in Developing Countries: A Framework with Examples from East Asia. World Development. https://doi.org/10.1016/50305750X(98)00071-0

Lander, E. (2018). El Estado Mágico Sigue Ahí, las Continuidades y Rupturas en la Historia del Petroestado venezolano. Nueva Sociedad, 274, 30-43.

Lewis, W. A. (1954). Economic Development with Unlimited Supplies of Labour. The Manchester School.

Lin, J. (2004). Lessons of China's Transition from a Planned Economy to a Market Economy. Peking University and Hong Kong University of Science and Technology.

Lin, J. y Li, Zhou. (2013). The China Miracle: Development Strategy and Economic Reform. The Australian Economic Review, 46(3), 259-68.

Lin, J..y Monga, C. (2010). Growth Identification and Facilitation, The Role of the State in the Dynamics of Structural Change". Working paper. The World Bank Development Economics Office of the Vice President.

Lopez, M. (2006). Venezuela 2001-2004: actores y estrategias en la lucha hegemónica. CLACSO.

Lucena, H. y Carmona, H. (2011). La Industria Venezolana, Auge y Ocaso a Través de Tres Modelos Productivos. Ensayos de Economía, 39.

Murphy, K., Shleifer, A. y Vishny, R. (1989). Industrialization and the Big Push. The Journal of Political Economy, 97(5), 1003-1026.

Naciones Unidas. (2018). Informe del Experto Independiente sobre la promoción de un orden internacional democrático y equitativo acerca de su misión a la República Bolivariana de Venezuela y al Ecuador. Consejo de Derechos Humanos.

Naudé, W. y Szirmai, A. (2012). The importance of manufacturing in economic development: Past, present and future perspectives.

Ocampo, J. (2014). Latin American Structuralism and Production Development Strategies

Ocampo, J. 2005. The Quest for Dynamic Efficiency: Structural Dynamics and Economic Growth in Developing Countries. Beyond Reforms, Structural Dynamics and Macroeconomic Vulnerability.

Solow, R. (1956). A Contribution to the Theory of Economic Growth. The Quarterly Journal of Economics, 70(1), 65-94

Tapia, J. y Astarita, R. (2011). La Gran Recesión y el Capitalismo del Siglo XXI. Editorial Los Libros de la Catarata. Madrid, España.

The Conference Board Total Economy Database ${ }^{\mathrm{TM}}$ (2018). https://www.conferenceboard.org/data/economydatabase/index.cfm?id=27762 
Timmer, M. de Vries, Gaaitzen. J., \& de Vries, Klaas. (2015). "Patterns of Structural Change in Developing Countries." . In J. Weiss, \& M. Tribe (Eds.), Routledge Handbook of Industry and Development. Routledge.

Transparencia Venezuela, (s.f.) Informe Empresas Propiedad del Estado Venezolano, Séptima Parte. En: Transparencia Venezuela. P.22

U.S. Department of the Treasury. (2019). Treasury Increases Preassure on Cuba to End Support to Maduro by Imposing further Oil Sector Sanctions

Vera, L. (2018). ¿Cómo Explicar la Catástrofe Económica de Venezuela? Nueva Sociedad, 274, 8396

White House Office of Trade and Manufacturing Policy. (2018). How China's Economic Aggression Threatens the Technologies and Intellectual Property of the United States and the World. The White House. https://www.whitehouse.gov/wP-content/uploads/2018/06/FINAL-ChinaTechnology-Report-6.18.18-PDF.pdf.

Worldsteel

Association.

Recuperado

de:

https://web.archive.org/web/20150420161500/http://www.worldsteel.org/dms/internetDocume ntList/statistics-archive/production-archive/steel-archive/steel-annually/steel-annually-19802013/document/steel\%20annually\%201980-2013.pdf

Zhu, X. (2012). Understanding China's Growth: Past, Present and Future. Journal of Economic Perspective, 26(4), 103-124 